\title{
STABILITY ANALYSIS OF LINEAR NEUTRAL SYSTEMS WITH MULTIPLE TIME DELAYS
}

\author{
KEYUE ZHANG
}

Received 25 August 2004 and in revised form 25 October 2004

This paper studies the asymptotic stability of linear neutral systems with multiple time delays. Using the characteristic equation of the system, new delay-independent stability criteria are derived in terms of the spectral radius of modulus matrices. Numerical examples are given to demonstrate the validity of our new criteria.

\section{Introduction}

Mathematical models with time delays are often encountered in various engineering systems due to measurement and computational delays, transmission and transport lags. Since the existence of time delays is frequently the source of instability, an important theme in neutral delay-differential systems is the stability of response characteristics.

Different methods have been presented to deal with the stability problem of neutral systems with time delays in the literature. A number of stability criteria based on the characteristic equation approach, involving the determination of eigenvalues, measures and norms of matrices, or matrix conditions in terms of Hurwitz matrices, have been presented by Hale et al. [4], $\mathrm{Li}$ [9], Hu et al. [6], and Cao and $\mathrm{He}$ [1, 2]. Some stability criteria (delay-independent or delay-dependent) are given in terms of the Lyapunov function and matrix inequalities (see, e.g., Lien et al. [10], Fridman [3], and Niculescu [11]). Based on the linear matrix inequality (LMI) approach, robust stability conditions have been developed to make the criteria less conservative, see, for example, Park [12].

Recently, the study of stability has been extended to neutral systems with multiple time delays. By making use of the characteristic equation of the system, Hui and $\mathrm{Hu}$ [7] derived a delay-independent stability criterion in terms of the matrix measure and spectral norm of the matrix. In order to reduce the conservatism in the criterion of Hui and $\mathrm{Hu}$ [7], Won and Park [13] proposed a new delay-independent criterion in terms of the spectral radius of modulus matrices. However, we have found that a technical error, as shown in the next section, exists in the proof of the criterion of Won and Park [13].

This paper deals with the asymptotic stability of linear neutral systems with multiple time delays. Using the characteristic equation of the system, new delay-independent stability criteria are derived. Scalar inequalities involving the spectral radius and modulus 
Table 2.1

\begin{tabular}{ll}
\hline $\mathbb{R}^{n}\left(\mathbb{C}^{n}\right)$ & $n$-dimensional real (complex) space \\
$\mathbb{R}^{n \times n}\left(\mathbb{C}^{n \times n}\right)$ & Set of all real (complex) $n \times n$ matrices \\
$I$ & Unit matrix \\
$\lambda_{j}(A)$ & $j$ th eigenvalue of matrix $A$ \\
$\lambda_{\max }(A)$ & Maximum eigenvalue of matrix $A$ \\
$A^{T}$ & Transpose of matrix $A$ \\
$A^{*}$ & Conjugate transpose of matrix $A$ \\
$\operatorname{det}(A)$ & Determinant of matrix $A$ \\
$\operatorname{Re}(s)$ & Real part of complex number $s$ \\
$\rho(A)$ & Spectral radius of matrix $A$ \\
$|s|$ & Modulus of complex number $s$ \\
$|A|$ & Modulus matrix of matrix $A ;|A|=\left[\left|a_{i j}\right|\right]$ with $A=\left[a_{i j}\right]$ \\
$A \leq B$ & $a_{i j} \leq b_{i j}$ with $A=\left[a_{i j}\right]$ and $B=\left[b_{i j}\right]$ \\
$\|A\|$ & Spectral norm of matrix $A ;\|A\|=\sqrt{\lambda_{\max }\left(A^{*} A\right)}$ \\
$\mu(A)$ & Matrix measure of matrix $A ; \mu(A)=\frac{1}{2} \lambda_{\max }\left(A+A^{*}\right)$ \\
\hline
\end{tabular}

matrices constitute the mathematical foundations of our approach. Numerical examples are given to demonstrate the validity of our new criteria and to compare them with the existing ones.

\section{System description and previous results}

Throughout this paper, the conventions in Table 2.1 are used.

Consider the following linear neutral system with multiple time delays:

$$
\dot{x}(t)=A x(t)+\sum_{j=1}^{m}\left[B_{j} x\left(t-\tau_{j}\right)+C_{j} \dot{x}\left(t-\tau_{j}\right)\right],
$$

where $x(t) \in \mathbb{C}^{n \times 1}$ is the state vector, the constant parameters $\tau_{j} \geq 0$ with $\tau=\max \left\{\tau_{j}, j=\right.$ $1,2, \ldots, m\}$ represent the delay arguments, $A, B_{j}$, and $C_{j} \in \mathbb{C}^{n \times n}(j=1,2, \ldots, m)$, and the system matrix $A$ is assumed to be a Hurwitz matrix, that is, all the eigenvalues of $A$ have negative real parts.

For simplicity, the following notations defined in Won and Park [13] are employed:

$$
\begin{gathered}
B(s)=\sum_{j=1}^{m} B_{j} \exp \left(-s \tau_{j}\right), \quad C(s)=\sum_{j=1}^{m} C_{j} \exp \left(-s \tau_{j}\right), \\
B_{m}=\sum_{j=1}^{m}\left|B_{j}\right|, \quad C_{m}=\sum_{j=1}^{m}\left|C_{j}\right|, \quad C_{A m}=\sum_{j=1}^{m}\left|C_{j} A\right|, \quad c=\sum_{j=1}^{m}\left\|C_{j}\right\| .
\end{gathered}
$$


The characteristic equation of the neutral system (2.1) is described by

$$
P(s)=\operatorname{det}[s I-A-B(s)-s C(s)]=0 .
$$

Here $P(s)$ denotes the characteristic function. The following three lemmas are cited and will be used in the proof of our main results.

Lemma 2.1 (Hale and Verduyn Lunel [5]). If $a_{D}=\sup \{\operatorname{Re}(s): P(s)=0\}$ and $a_{D}<0$, then the neutral system (2.1) is asymptotically stable.

Lemma 2.2 (Lancaster and Tismenetsky [8]). Let $R \in \mathbb{C}^{n \times n}$. If $\rho(R)<1$, then $(I-R)^{-1}$ exists, $\operatorname{det}(I \pm R) \neq 0$ and

$$
(I-R)^{-1}=I+R+R^{2}+\cdots .
$$

Lemma 2.3 (Lancaster and Tismenetsky [8]). Let $R, T$, and $V \in \mathbb{C}^{n \times n}$. If $|R| \leq V$, then

(a) $|R T| \leq|R||T| \leq V|T|$,

(b) $|R+T| \leq|R|+|T| \leq V+|T|$,

(c) $\rho(R) \leq \rho(|R|) \leq \rho(V)$,

(d) $\rho(R T) \leq \rho(|R||T|) \leq \rho(V|T|)$,

(e) $\rho(R+T) \leq \rho(|R+T|) \leq \rho(|R|+|T|) \leq \rho(V+|T|)$.

Based on the characteristic equation (2.3), Hui and $\mathrm{Hu}$ [7] presented the following theorem.

Theorem 2.4 (Hui and $\mathrm{Hu}$ [7]). The neutral delay-differential system (2.1) is asymptotically stable if $c<1$ and

$$
\mu(A)+\sum_{j=1}^{m}\left\|B_{j}\right\|+\frac{1}{1-c}\left[\sum_{j=1}^{m}\left\|C_{j} A\right\|+\sum_{j, k=1}^{m}\left\|C_{j} B_{k}\right\|\right]<0 .
$$

Obviously, the condition $\mu(A)<0$ is necessary to satisfy sufficient condition (2.5) and is a strict restriction for application. To reduce the conservatism, Won and Park [13] derived the following theorem in terms of the spectral radius of the matrix which is the combination of the modulus matrices.

Theorem 2.5 (Won and Park [13]). The neutral delay-differential system (2.1) is asymptotically stable if $c<1$ and

$$
\rho\left[F_{m}\left(B_{m}+\frac{C_{A m}+C_{m} B_{m}}{1-c}\right)\right]<1,
$$

where $F_{m}$ denotes a matrix formed by taking the maximum magnitude of each element of $F(s)=(s I-A)^{-1}$ for $\operatorname{Re}(s)>0$.

The numerical example given in Won and Park [13] showed that the condition in Theorem 2.5 is less conservative than that in Theorem 2.4. Unfortunately, there exists a technical error in the proof of Theorem 2.5 (Won and Park [13, Theorem 1]). In fact, it 
178 Stability criteria of linear neutral systems

is easy to see that, in general, the inequality

$$
\rho(|R||T|) \leq \rho(\|R\||T|)
$$

does not hold for given matrices $R$ and $T$. Thus, in general, the following inequality

$$
\begin{aligned}
& \rho\left[F_{m}\left(B_{m}+\left|I+C(s)+C^{2}(s)+\cdots\right|(|C(s) A|+|C(s) B(s)|)\right)\right] \\
& \quad \leq \rho\left[F_{m}\left(B_{m}+|| I+C(s)+C^{2}(s)+\cdots||(|C(s) A|+|C(s) B(s)|)\right)\right]
\end{aligned}
$$

does not hold. Therefore, additional prerequisites might be required for the proof of Theorem 2.5.

\section{Main results}

We define

$$
C_{B m 1}=\sum_{j=1}^{m} \sum_{k \geq j}^{m}\left|C_{j} B_{k}+C_{k} B_{j}\left(1-\delta_{j k}\right)\right|, \quad C_{B m 2}=\sum_{j, k=1}^{m}\left|C_{j} B_{k}\right|,
$$

where $\delta_{j k}$ is the Dirac $\delta$-function.

Theorem 3.1. The neutral delay-differential system (2.1) is asymptotically stable if $\rho\left(C_{m}\right)<$ 1 and

$$
\rho\left[F_{m}\left(B_{m}+\left(I-C_{m}\right)^{-1}\left(C_{A m}+C_{B m 1}\right)\right)\right]<1 .
$$

Proof. For $\operatorname{Re}(s) \geq 0$, in view of

$$
|C(s)|=\left|\sum_{j=1}^{m} C_{j} \exp \left(-s \tau_{j}\right)\right| \leq \sum_{j=1}^{m}\left|C_{j} \exp \left(-s \tau_{j}\right)\right| \leq \sum_{j=1}^{m}\left|C_{j}\right|=C_{m},
$$

it follows from Lemma 2.2 that $(I-C(s))^{-1}$ exists and $\operatorname{det}[I-C(s)] \neq 0$.

According to Lemma 2.1, system (2.1) is asymptotically stable if

$$
\operatorname{det}[s I-A-B(s)-s C(s)] \neq 0, \quad \text { for } \operatorname{Re}(s) \geq 0 .
$$

Since $\operatorname{det}[I-C(s)] \neq 0,(3.4)$ is equivalent to

$$
\operatorname{det}\left[s I-(I-C(s))^{-1}(A+B(s))\right] \neq 0, \quad \text { for } \operatorname{Re}(s) \geq 0 .
$$

Employing the well-known relation $(I-C(s))^{-1}=I+(I-C(s))^{-1} C(s)$, we have

$$
\begin{aligned}
\operatorname{det}[s I & \left.-(I-C(s))^{-1}(A+B(s))\right] \\
& =\operatorname{det}\left[s I-\left(I-(I-C(s))^{-1} C(s)\right)(A+B(s))\right] \\
& =\operatorname{det}\left[s I-A-B(s)-(I-C(s))^{-1}(C(s) A+C(s) B(s))\right] \\
& =\operatorname{det}(s I-A) \operatorname{det}\left[I-F(s)\left(B(s)+(I-C(s))^{-1}(C(s) A+C(s) B(s))\right)\right],
\end{aligned}
$$


where $F(s)=(s I-A)^{-1}$. Since $A$ is a Hurwitz matrix, $\operatorname{det}(s I-A) \neq 0$ for $\operatorname{Re}(s) \geq 0$. It follows from (3.4), (3.5), (3.6), and Lemma 2.2 that (2.1) is asymptotically stable if

$$
\rho\left[F(s)\left(B(s)+(I-C(s))^{-1}(C(s) A+C(s) B(s))\right)\right]<1, \quad \text { for } \operatorname{Re}(s) \geq 0 .
$$

According to Lemma 2.3, for $\operatorname{Re}(s) \geq 0$, the following relations can be easily obtained:

$$
\begin{aligned}
|B(s)| & =\left|\sum_{j=1}^{m} B_{j} \exp \left(-s \tau_{j}\right)\right| \leq \sum_{j=1}^{m}\left|B_{j} \exp \left(-s \tau_{j}\right)\right| \leq \sum_{j=1}^{m}\left|B_{j}\right|=B_{m}, \\
|C(s) A| & =\left|\sum_{j=1}^{m} C_{j} A \exp \left(-s \tau_{j}\right)\right| \leq \sum_{j=1}^{m}\left|C_{j} A \exp \left(-s \tau_{j}\right)\right| \leq \sum_{j=1}^{m}\left|C_{j} A\right|=C_{A m}, \\
|C(s) B(s)| & =\left|\sum_{j=1}^{m} \sum_{k=1}^{m} C_{j} B_{k} \exp \left(-s\left(\tau_{j}+\tau_{k}\right)\right)\right| \\
& =\left|\sum_{j=1}^{m} C_{j} B_{j} \exp \left(-s \tau_{j}\right)+\sum_{j=1}^{m} \sum_{k>j}^{m}\left(C_{j} B_{k}+C_{k} B_{j}\right) \exp \left(-s\left(\tau_{j}+\tau_{k}\right)\right)\right| \\
& =\sum_{j=1}^{m}\left|C_{j} B_{j} \exp \left(-s \tau_{j}\right)\right|+\sum_{j=1}^{m} \sum_{k>j}^{m}\left|\left(C_{j} B_{k}+C_{k} B_{j}\right) \exp \left(-s\left(\tau_{j}+\tau_{k}\right)\right)\right| \\
& \leq \sum_{j=1}^{m} \sum_{k \geq j}^{m}\left|C_{j} B_{k}+C_{k} B_{j}\left(1-\delta_{j k}\right)\right|=C_{B m 1} .
\end{aligned}
$$

Moreover, using (3.3) and Lemmas 2.2 and 2.3, we have for $\operatorname{Re}(s) \geq 0$,

$$
\begin{aligned}
\left|(I-C(s))^{-1}\right| & =\left|I+C(s)+C^{2}(s)+\cdots\right| \\
& \leq I+|C(s)|+\left|C^{2}(s)\right|+\cdots \\
& \leq I+C_{m}+C_{m}^{2}+\cdots \\
& =\left(I-C_{m}\right)^{-1} .
\end{aligned}
$$

Now, using Lemma 2.3, together with (3.3), (3.8), (3.9),(3.10), and (3.11), we can obtain for $\operatorname{Re}(s) \geq 0$,

$$
\begin{aligned}
\rho[F(s) & \left.\left(B(s)+(I-C(s))^{-1}(C(s) A+C(s) B(s))\right)\right] \\
& \leq \rho\left[|F(s)|\left(|B(s)|+\left|(I-C(s))^{-1}(C(s) A+C(s) B(s))\right|\right)\right] \\
& \leq \rho\left[|F(s)|\left(|B(s)|+\left|(I-C(s))^{-1}\right|(|C(s) A|+|C(s) B(s)|)\right)\right] \\
& \leq \rho\left[F_{m}\left(B_{m}+\left(I-C_{m}\right)^{-1}\left(C_{A m}+C_{B m 1}\right)\right)\right] .
\end{aligned}
$$

Therefore, condition (3.2) implies that (3.7) holds. The proof is completed.

Corollary 3.2. The neutral delay-differential system (2.1) is asymptotically stable if $\rho\left(C_{m}\right)$ $<1$ and

$$
\rho\left[F_{m}\left(B_{m}+\left(I-C_{m}\right)^{-1}\left(C_{A m}+C_{B m 2}\right)\right)\right]<1 .
$$


Proof. Taking notice of

$$
\begin{aligned}
C_{B m 1} & =\sum_{j=1}^{m} \sum_{k \geq j}^{m}\left|C_{j} B_{k}+C_{k} B_{j}\left(1-\delta_{j k}\right)\right| \\
& \leq \sum_{j=1}^{m} \sum_{k \geq j}^{m}\left|C_{j} B_{k}\right|+\left|C_{k} B_{j}\left(1-\delta_{j k}\right)\right|=\sum_{j, k=1}^{m}\left|C_{j} B_{k}\right|=C_{B m 2},
\end{aligned}
$$

we have from Lemma 2.3(a)

$$
\rho\left[F_{m}\left(B_{m}+\left(I-C_{m}\right)^{-1}\left(C_{A m}+C_{B m 1}\right)\right)\right] \leq \rho\left[F_{m}\left(B_{m}+\left(I-C_{m}\right)^{-1}\left(C_{A m}+C_{B m 2}\right)\right)\right]<1 .
$$

The result follows from Theorem 3.1. This proves the corollary.

Moreover, taking notice of

$$
C_{B m 2}=\sum_{j, k=1}^{m}\left|C_{j} B_{k}\right| \leq \sum_{j=1}^{m} \sum_{k=1}^{m}\left|C_{j}\right|\left|B_{k}\right|=\sum_{j=1}^{m}\left|C_{j}\right| \sum_{k=1}^{m}\left|B_{k}\right|=\sum_{j=1}^{m}\left|C_{j}\right| B_{m}=C_{m} B_{m},
$$

we obtain the following corollary.

COROLlary 3.3. The neutral delay-differential system (2.1) is asymptotically stable if $\rho\left(C_{m}\right)$ $<1$ and

$$
\rho\left[F_{m}\left(B_{m}+\left(I-C_{m}\right)^{-1}\left(C_{A m}+C_{m} B_{m}\right)\right)\right]<1 .
$$

\section{Illustrative examples}

Example 4.1. Consider the linear neutral system with multiple time delays

$$
\dot{x}(t)=A x(t)+B_{1} x\left(t-\tau_{1}\right)+C_{1} \dot{x}\left(t-\tau_{1}\right)+B_{2} x\left(t-\tau_{2}\right)+C_{2} \dot{x}\left(t-\tau_{2}\right),
$$

where $\tau_{1}>0$ and $\tau_{2}>0$ are constants,

$$
\begin{array}{cc}
A=\left[\begin{array}{cc}
-3 & -2 \\
1 & 0
\end{array}\right], \quad B_{1}=\alpha\left[\begin{array}{cc}
0.2 & 0.1 \\
-0.1 & 0.2
\end{array}\right], \quad C_{1}=\left[\begin{array}{cc}
0.05 & 0.1 \\
0 & 0.1
\end{array}\right], \\
B_{2}=\alpha\left[\begin{array}{cc}
0.4 & -0.3 \\
-0.1 & -0.05
\end{array}\right], & C_{2}=\left[\begin{array}{cc}
0 & 0.1 \\
0.05 & 0
\end{array}\right],
\end{array}
$$

and $\alpha$ is a nonzero constant.

Since the system matrix $A$ is Hurwitz, the stability bounds can be calculated in terms of $\alpha$ by using our new criteria. The rational function matrix $F(s)$ is (Won and Park [13])

$$
F(s)=\frac{1}{s^{2}+3 s+2}\left[\begin{array}{cc}
s & -2 \\
1 & s+3
\end{array}\right]
$$


The modulus matrices are easily computed as

$$
\begin{gathered}
F_{m}=\left[\begin{array}{cc}
0.3333 & 1 \\
0.5 & 1.5
\end{array}\right], \quad B_{m}=|\alpha|\left[\begin{array}{cc}
0.6 & 0.4 \\
0.2 & 0.25
\end{array}\right], \quad C_{m}=\left[\begin{array}{ll}
0.05 & 0.2 \\
0.05 & 0.1
\end{array}\right], \\
C_{A m}=\left[\begin{array}{cc}
0.15 & 0.1 \\
0.25 & 0.1
\end{array}\right], \quad C_{B m 1}=|\alpha|\left[\begin{array}{cc}
0.01 & 0.03 \\
0.03 & 0.035
\end{array}\right], \quad C_{B m 2}=|\alpha|\left[\begin{array}{cc}
0.03 & 0.07 \\
0.05 & 0.045
\end{array}\right] .
\end{gathered}
$$

Then, the stability bounds can be obtained as

$$
\begin{aligned}
& \text { Theorem } 3.1:|\alpha|<0.3595, \\
& \text { Corollary } 3.2:|\alpha|<0.3364, \\
& \text { Corollary } 3.3:|\alpha|<0.3318
\end{aligned}
$$

As pointed out by Won and Park [13], the stability criteria derived by Hui and $\mathrm{Hu}$ [7] are not available because the matrix measure $\mu(A)=0.0811>0$.

Example 4.2. Consider the linear neutral system (4.1) with

$$
\begin{gathered}
A=\left[\begin{array}{cc}
-2 & 1 \\
-1 & -1
\end{array}\right], \quad B_{1}=\alpha\left[\begin{array}{cc}
0.275 & -0.1125 \\
-0.35 & 0.325
\end{array}\right], \quad C_{1}=\left[\begin{array}{cc}
0.1 & 0.05 \\
0 & 0.1
\end{array}\right], \\
B_{2}=\alpha\left[\begin{array}{cc}
0.4 & -0.3 \\
-0.1 & -0.05
\end{array}\right], \quad C_{2}=\left[\begin{array}{cc}
0 & 0.1 \\
0.1 & 0.05
\end{array}\right]
\end{gathered}
$$

Since the system matrix $A$ is Hurwitz, $F_{m}$ can be obtained for some $s$ on imaginary axis by the maximum modulus theorem. The modulus matrices are computed as

$$
\begin{gathered}
F_{m}=\frac{1}{3}\left[\begin{array}{cc}
1.1961 & 1 \\
1 & 2
\end{array}\right], \quad B_{m}=|\alpha|\left[\begin{array}{cc}
0.675 & 0.4125 \\
0.45 & 0.375
\end{array}\right], \quad C_{m}=\left[\begin{array}{ll}
0.1 & 0.15 \\
0.1 & 0.15
\end{array}\right], \\
C_{A m}=\left[\begin{array}{ll}
0.35 & 0.15 \\
0.35 & 0.15
\end{array}\right], \quad C_{B m 1}=|\alpha|\left[\begin{array}{cc}
0.02 & 0.01 \\
0.07 & 0.065
\end{array}\right], \quad C_{B m 2}=|\alpha|\left[\begin{array}{ll}
0.09 & 0.075 \\
0.09 & 0.075
\end{array}\right] .
\end{gathered}
$$

Then, the stability bounds can be obtained as

Theorem $3.1:|\alpha|<0.5107$,

Corollary $3.2:|\alpha|<0.4658$,

Corollary $3.3:|\alpha|<0.4432$. 
Moreover, taking notice of

$$
\begin{aligned}
& \sum_{j=1}^{2}\left\|B_{j}\right\|=1.0578|\alpha|, \quad c=\sum_{j=1}^{2}\left\|C_{j}\right\|=0.2562, \\
& \sum_{j=1}^{2}\left\|C_{j} A\right\|=0.5389, \quad \sum_{j, k=1}^{2}\left\|C_{j} B_{k}\right\|=0.1917|\alpha|,
\end{aligned}
$$

we obtain

$$
\mu(A)+\sum_{j=1}^{2}\left\|B_{j}\right\|+\frac{1}{1-c}\left[\sum_{j=1}^{m}\left\|C_{j} A\right\|+\sum_{j, k=1}^{m}\left\|C_{j} B_{k}\right\|\right]=-0.2755+1.3156|\alpha| .
$$

Thus, the criterion presented by Hui and $\mathrm{Hu}[7]$ gives $|\alpha|<0.2094$. In this example, we can see that our results are less conservative than that given in the previous work.

\section{Conclusions}

In this paper, we have studied the stability of linear neutral systems with multiple time delays. Using the characteristic function, delay-independent stability criteria have been derived in terms of scalar inequalities involving the spectral radius of modulus matrices. A misleading statement in Won and Park [13] has been pointed out. Numerical examples are given to show that the new stability criteria are less conservative and more powerful compared to those in the literature.

\section{Acknowledgments}

This work is partly supported by the research grant from Emei School, Southwest Jiaotong University. The author is indebted to Professor Dengqing Cao for beneficial discussions on this work.

\section{References}

[1] D. Q. Cao and P. He, Stability criteria of linear neutral systems with a single delay, Appl. Math. Comput. 148 (2004), no. 1, 135-143.

[2] _ Sufficient conditions for stability of linear neutral systems with a single delay, Appl. Math. Lett. 17 (2004), no. 2, 139-144.

[3] E. Fridman, New Lyapunov-Krasovskii functionals for stability of linear retarded and neutral type systems, Systems Control Lett. 43 (2001), no. 4, 309-319.

[4] J. K. Hale, E. F. Infante, and F.-S. P. Tsen, Stability in linear delay equations, J. Math. Anal. Appl. 105 (1985), no. 2, 533-555.

[5] J. K. Hale and S. M. Verduyn Lunel, Introduction to Functional-Differential Equations, Applied Mathematical Sciences, vol. 99, Springer, New York, 1993.

[6] G.-D. Hu, G.-D. Hu, and B. Cahlon, Algebraic criteria for stability of linear neutral systems with a single delay, J. Comput. Appl. Math. 135 (2001), no. 1, 125-133.

[7] G.-D. Hui and G.-D. Hu, Simple criteria for stability of neutral systems with multiple delays, Internat. J. Systems Sci. 28 (1997), no. 12, 1325-1328.

[8] P. Lancaster and M. Tismenetsky, The Theory of Matrices, Computer Science and Applied Mathematics, Academic Press, Florida, 1985. 
[9] L. M. Li, Stability of linear neutral delay-differential systems, Bull. Austral. Math. Soc. 38 (1988), no. 3, 339-344.

[10] C.-H. Lien, K.-W. Yu, and J.-G. Hsieh, Stability conditions for a class of neutral systems with multiple time delays, J. Math. Anal. Appl. 245 (2000), no. 1, 20-27.

[11] S.-I. Niculescu, On delay-dependent stability under model transformations of some neutral linear systems, Internat. J. Control 74 (2001), no. 6, 609-617.

[12] J.-H. Park, A new delay-dependent criterion for neutral systems with multiple delays, J. Comput. Appl. Math. 136 (2001), no. 1-2, 177-184.

[13] S. Won and J.-H. Park, A note on the stability analysis of neutral systems with multiple timedelays, Internat. J. Systems Sci. 32 (2001), no. 4, 409-412.

Keyue Zhang: Department of Applied Mathematics and Mechanics, Southwest Jiaotong University, Emei Campus, Emeishan, Sichuan 614202, China

E-mail address: keyuezhang@yahoo.com.cn 


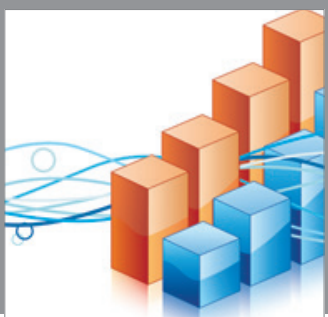

Advances in

Operations Research

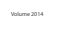

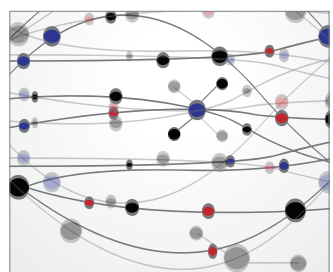

\section{The Scientific} World Journal
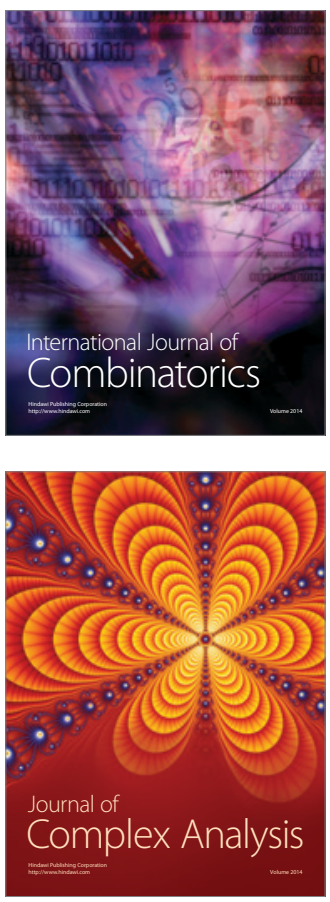

International Journal of

Mathematics and

Mathematical

Sciences
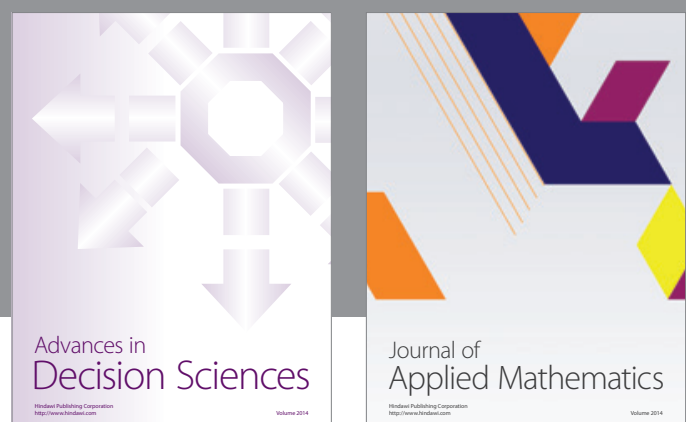

Journal of

Applied Mathematics
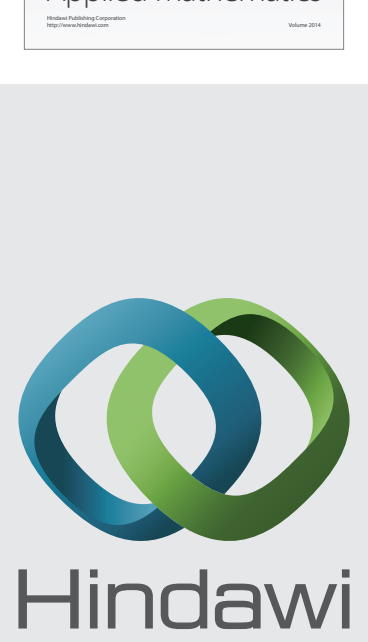

Submit your manuscripts at http://www.hindawi.com
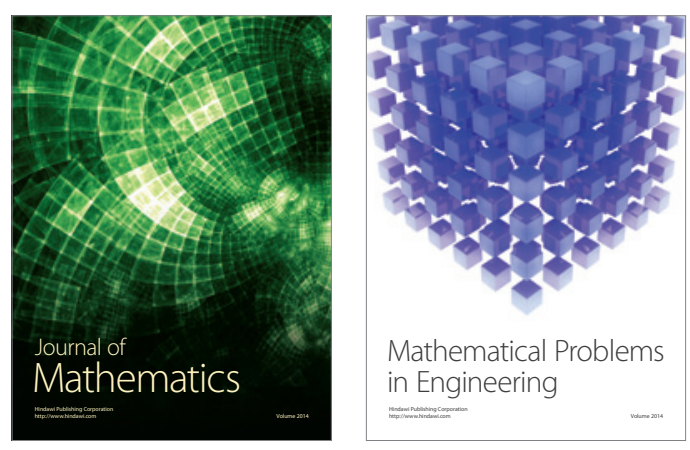

Mathematical Problems in Engineering
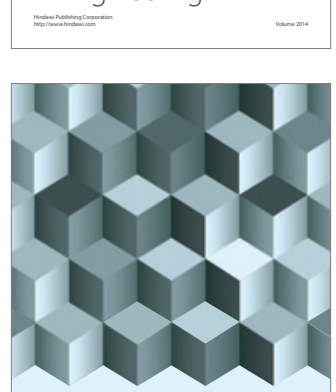

Journal of

Function Spaces
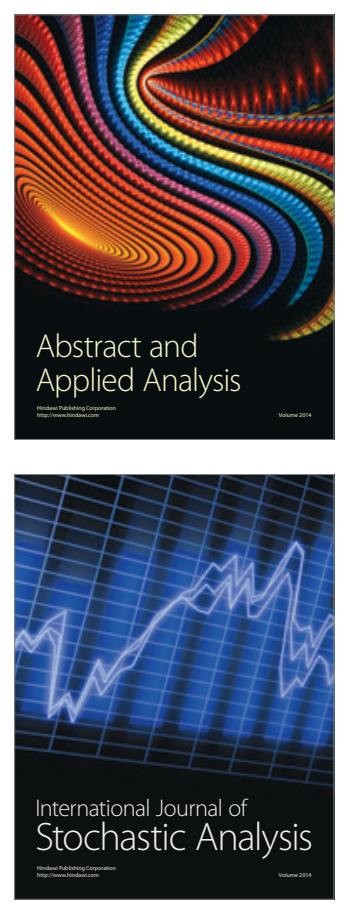

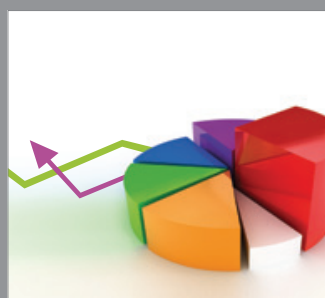

ournal of

Probability and Statistics

Promensencen
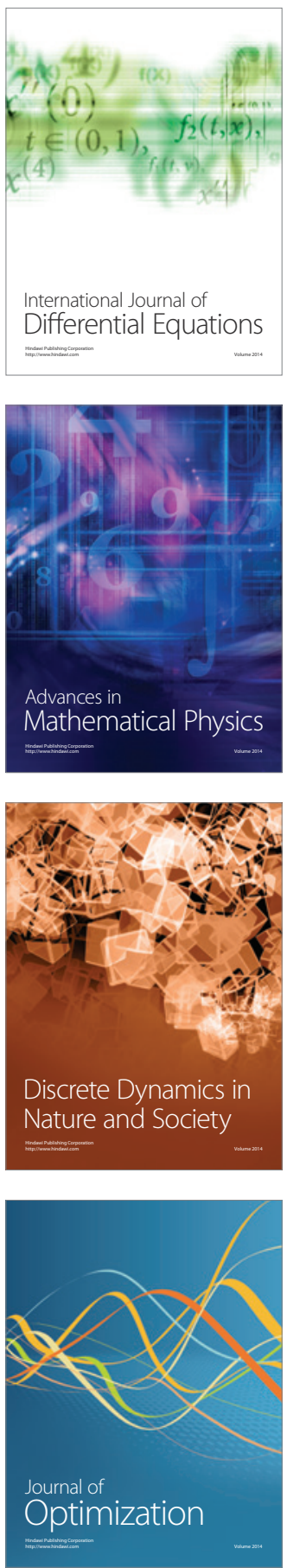